\title{
The Cauchy problem for the seventh-order dispersive equation in Sobolev space
}

Hongjun Wang ${ }^{1 *}$ and Yan Zheng ${ }^{2}$

${ }^{*}$ Correspondence: hsdwhj@163.com

${ }^{1}$ College of Mathematics and Information Science, Henan Normal University, Xinxiang, Henan 453007, P.R. China

Full list of author information is available at the end of the article

\section{Abstract}

This paper is devoted to the Cauchy problem for the higher-order dispersive equation $u_{t}+\partial_{x}^{7} u=\partial_{x}^{2}\left(u^{2}\right), x, t \in \mathbf{R}$. The local well-posedness of the associated Cauchy problem is established in Sobolev space $H^{5}(\mathbf{R})$ with $s>-\frac{7}{4}$ with the aid of the Fourier restriction norm method.

MSC: $35 \mathrm{~K} 30$

Keywords: Cauchy problem; well-posedness; Sobolev spaces

\section{Introduction}

In this paper, we are concerned with the Cauchy problem for the following seventh-order dispersive equation:

$$
\begin{aligned}
& u_{t}+\partial_{x}^{7} u=\partial_{x}^{2}\left(u^{2}\right), \quad x, t \in \mathbf{R}, \\
& u(x, 0)=u_{0}(x) .
\end{aligned}
$$

Kenig et al. [1] established that

$$
\begin{aligned}
& u_{t}+\partial_{x}^{2 j+1} u+P\left(u, \partial_{x} u, \ldots, \partial_{x}^{2 j} u\right)=0, \quad j \in \mathbf{N}, x, t \in \mathbf{R}, \\
& u(x, 0)=u_{0}(x),
\end{aligned}
$$

is locally well-posed in some weighted Sobolev spaces for small initial data and for arbitrary initial data. Recently, Pilod [2] studied the following higher-order nonlinear dispersive equation:

$$
u_{t}+\partial_{x}^{2 j+1} u=\sum_{0 \leq j_{1}+j_{2} \leq 2 j} a_{j_{1}, j_{2}} \partial_{x}^{j_{1}} u \partial_{x}^{j_{2}} u
$$

where $x, t \in \mathbf{R}$ and $u$ is a real- (or complex-) valued function and proved it is locally wellposed in weighted Besov and Sobolev spaces for small initial data and proved ill-posedness results when $a_{0, k} \neq 0$ for some $k>j$ in the sense that (1.5) cannot have its flow map $C^{2}$ at the origin in $H^{s}(R)$. Very recently, Guo et al. [3] studied the Cauchy problem for

$$
u_{t}+\partial_{x}^{5}+c_{1} \partial_{x} u \partial_{x}^{2} u+c_{2} u \partial_{x}^{3} u=0
$$


and he proved that it is locally well-posed in $H^{s}(\mathbf{R})$ with $s \geq \frac{5}{4}$ with the aid of a short time Bourgain space.

In this paper, inspired by [1-5], by using the Fourier restriction norm method, we establish that (1.1)-(1.2) is locally well-posed in Sobolev space $H^{s}$ with $s>-\frac{7}{4}$.

Now we give some notations and definitions. Throughout this paper, we always assume that $\psi$ is a smooth function, $\psi_{\delta}(t)=\psi\left(\frac{t}{\delta}\right)$, satisfying $0 \leq \psi \leq 1, \psi=1$ when $t \in[0,1]$, $\operatorname{supp} \psi \subset[-1,2]$ and $\sigma=\tau-\xi^{7}, \sigma_{k}=\tau_{k}-\xi_{k}^{7}(k=1,2)$,

$$
\begin{aligned}
W(t) u_{0} & =\int_{\mathbf{R}} e^{i\left(x \xi-t \xi^{7}\right)} \mathscr{F}_{x} u_{0}(\xi) d \xi, \\
\|f\|_{L_{t}^{q} L_{x}^{p}} & =\left(\int_{\mathbf{R}}\left(\int_{\mathbf{R}}|f(x, t)|^{p} d x\right)^{\frac{q}{p}} d t\right)^{\frac{1}{q}}, \quad\|f\|_{L_{t}^{p} L_{x}^{p}}=\|f\|_{L_{x t}^{p}} .
\end{aligned}
$$

$\langle\xi\rangle^{s}=\left(1+\xi^{2}\right)^{\frac{s}{2}}$ for any $\xi \in \mathbf{R}$, and $\mathscr{F} u$ denotes the Fourier transformation of $u$ with respect to its all variables. $\mathscr{F}^{-1} u$ denotes the Fourier inverse transformation of $u$ with respect to its all variables. $\mathscr{F}_{x} u$ denotes the Fourier transformation of $u$ with respect to its space variable. $\mathscr{F}_{x}^{-1} u$ denotes the Fourier inverse transformation of $u$ with respect to its space variable. $\mathscr{S}\left(\mathbf{R}^{n}\right)$ is the Schwarz space and $\mathscr{S}^{\prime}\left(\mathbf{R}^{n}\right)$ is its dual space. $H^{s}(\mathbf{R})$ is the Sobolev space with norm $\|f\|_{H^{s}(\mathbf{R})} \triangleq\left\|\langle\xi\rangle^{s} \mathscr{F}_{x} f\right\|_{L_{\xi}^{2}(\mathbf{R})}$. For any $s, b \in \mathbf{R}, X_{s, b}\left(\mathbf{R}^{2}\right)$ is the Bourgain space with phase function $\phi(\xi)=-\xi^{7}$. That is, a function $u(x, t)$ in $\mathscr{S}^{\prime}\left(\mathbf{R}^{2}\right)$ belongs to $X_{s, b}\left(\mathbf{R}^{2}\right)$ iff

$$
\|u\|_{X_{s, b}\left(\mathbf{R}^{2}\right)} \triangleq\left\|\langle\xi\rangle^{s}\langle\sigma\rangle^{b} \mathscr{F} u(\xi, \tau)\right\|_{L_{\tau}^{2}(\mathbf{R}) L_{\xi}^{2}(\mathbf{R})}<\infty
$$

For any given interval $L, X_{s, b}(\mathbf{R} \times L)$ is the space of the restriction of all functions in $X_{s, b}\left(\mathbf{R}^{2}\right)$ on $\mathbf{R} \times L$, and for $u \in X_{s, b}(\mathbf{R} \times L)$ its norm is

$$
\|u\|_{X_{s, b}(\mathbf{R} \times L)}=\inf \left\{\|U\|_{X_{s, b}\left(\mathbf{R}^{2}\right)} ;\left.U\right|_{\mathbf{R} \times L}=u\right\} .
$$

When $L=[0, T], X_{s, b}(\mathbf{R} \times L)$ is abbreviated as $X_{s, b}^{T}$.

The main result of this paper is as follows.

Theorem 1.1 Assume that $u_{0}(x) \in H^{s}(\mathbf{R})$ with $s>-\frac{7}{4}$. Then the Cauchy problem for (1.1) is locally well-posed.

The remainder of paper is arranged as follows. In Section 2, we make some preliminaries. In Section 3, we give an important bilinear estimate. In Section 4, we establish Theorem 1.1.

\section{Preliminaries}

Lemma 2.1 Let $b>\frac{1}{2}$. Then

$$
\begin{aligned}
& \|u\|_{L_{x t}^{4}} \leq C\|u\|_{X_{0, \frac{4 b}{7}}}, \\
& \left\|D_{x}^{\frac{5}{4}} u\right\|_{L_{t}^{4} L_{x}^{\infty}} \leq C\|u\|_{X_{0, b}}, \\
& \|u\|_{L_{t}^{4} L_{x}^{2}} \leq C\|u\|_{X_{0, \frac{1}{2} b}},
\end{aligned}
$$




$$
\begin{aligned}
& \|u\|_{X_{0,-\frac{1}{2} b}} \leq C\|u\|_{L_{t}^{\frac{4}{3}} L_{x}^{2}}, \\
& \left\|D_{x}^{\frac{5}{8}} u\right\|_{L_{x t}^{4}} \leq C\|u\|_{X_{0, \frac{3}{4} b}} .
\end{aligned}
$$

Proof For the proof of (2.1)-(2.5), we refer the readers to Lemma 2.1 of [5].

We have completed the proof of Lemma 2.1.

Lemma 2.2 Assume that $b=\frac{1}{2}+\epsilon$. Then

$$
\left\|I^{\frac{1}{2}}\left(u_{1}, u_{2}\right)\right\|_{L_{x t}^{2}} \leq C \prod_{k=1}^{2}\left\|u_{k}\right\|_{X_{0, b}}
$$

where

$$
\mathscr{F} I^{\frac{1}{2}}\left(u_{1}, u_{2}\right)(\xi, \tau)=\int_{\substack{\xi=\xi_{1}+\xi_{2} \\ \tau=\tau_{1}+\tau_{2}}}\left|\xi_{1}^{6}-\xi_{2}^{6}\right|^{\frac{1}{2}} \mathscr{F} u_{1}\left(\xi_{1}, \tau_{1}\right) \mathscr{F} u_{2}\left(\xi_{2}, \tau_{2}\right) d \xi_{1} d \tau_{1} .
$$

Lemma 2.2 is the case of $n=3$ of Lemma 3.1 of [5].

Lemma 2.3 For any $0<\delta<\frac{1}{2}$, and $s \in \mathbf{R}$, for $b>0$, we have

$$
\left\|\psi_{\delta}(t) W(t) u_{0}\right\|_{X_{s, b}} \leq C \delta^{\frac{1}{2}-b}\left\|u_{0}\right\|_{H^{s}}
$$

For $-\frac{1}{2}<b^{\prime} \leq 0 \leq b^{\prime}+1$, we have

$$
\left\|\psi_{\delta}(t) \int_{0}^{t} W(t-\tau) u d \tau\right\|_{X_{s, b}} \leq C \delta^{1+b^{\prime}-b}\|u\|_{X_{s, b^{\prime}}} .
$$

Lemma 2.3 can be found as Lemma 2.4 of [6].

\section{Bilinear estimates}

In this section, we will give an important bilinear estimate.

We give an important relation before proving the bilinear estimate.

$$
\left|\sigma-\sigma_{1}-\sigma_{2}\right|=\left|\xi^{7}-\xi_{1}^{7}-\xi_{2}^{7}\right| \sim \xi_{\min } \xi_{\max }^{6}
$$

where

$$
\begin{aligned}
& \xi_{\min }=\min \left\{|\xi|,\left|\xi_{1}\right|,\left|\xi_{2}\right|\right\} \\
& \xi_{\max }=\max \left\{|\xi|,\left|\xi_{1}\right|,\left|\xi_{2}\right|\right\} .
\end{aligned}
$$

Lemma 3.1 Let $s \geq-\frac{7}{4}+21 \epsilon, b=\frac{1}{2}+\epsilon$, where $0 \ll \epsilon \leq 1, b^{\prime}=-\frac{1}{2}+2 \epsilon$. Then

$$
\left\|\partial_{x}^{2} \prod_{k=1}^{2}\left(u_{k}\right)\right\|_{X_{s, b^{\prime}}} \leq C \prod_{k=1}^{2}\left\|u_{k}\right\|_{X_{s, b}} .
$$


Proof Let

$$
\begin{aligned}
& F_{k}\left(\xi_{k}, \tau_{k}\right)=\left\langle\xi_{k}\right\rangle^{s}\left\langle\sigma_{k}\right\rangle^{b} \mathcal{F} u_{k}\left(\xi_{k}, \tau_{k}\right), \\
& F(\xi, \tau)=\langle\xi\rangle^{-s}\langle\sigma\rangle^{b^{\prime}} \mathcal{F} u(\xi, \tau), \\
& \sigma=\tau-\xi^{7}, \quad \sigma_{k}=\tau_{k}-\xi_{k}^{7}, \quad k=1,2 .
\end{aligned}
$$

To establish (3.2), it is sufficient to derive the following inequality:

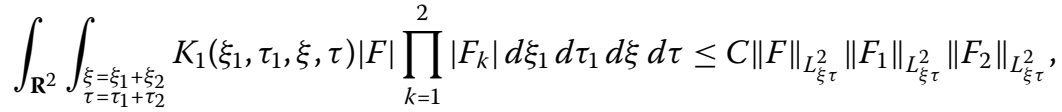

where

$$
K_{1}\left(\xi_{1}, \tau_{1}, \xi, \tau\right)=\frac{|\xi|^{2}\langle\sigma\rangle^{b^{\prime}}\langle\xi\rangle^{s}}{\prod_{k=1}^{2}\left\langle\xi_{k}\right\rangle^{s}\left\langle\sigma_{k}\right\rangle^{b}}
$$

Without loss of generality, we assume that $F \geq 0, F_{k} \geq 0(k=1,2)$. To derive (3.3), it suffices to prove that

$$
\int_{\mathbf{R}^{2}} \int_{\substack{\xi=\xi_{1}+\xi_{2} \\ \tau=\tau_{1}+\tau_{2}}} K_{1}\left(\xi_{1}, \tau_{1}, \xi, \tau\right) F \prod_{k=1}^{2} F_{k} d \xi_{1} d \tau_{1} d \xi d \tau \leq C\|F\|_{L_{\xi \tau}^{2}}\left\|F_{1}\right\|_{L_{\xi \tau}^{2}}\left\|F_{2}\right\|_{L_{\xi \tau}^{2}} .
$$

By using the symmetry between $\left|\xi_{1}\right|$ and $\xi_{2}$, without loss of generality, we can assume that $\left|\xi_{1}\right| \leq\left|\xi_{2}\right|$. Obviously,

$$
\left\{\xi=\xi_{1}+\xi_{2}, \tau=\tau_{1}+\tau_{2},\left|\xi_{2}\right| \geq\left|\xi_{1}\right|\right\} \subset \bigcup_{k=1}^{6} \Omega_{k},
$$

where

$$
\begin{aligned}
\Omega_{1}= & \left\{\left(\xi_{1}, \tau_{1}, \xi, \tau\right) \in \mathbf{R}^{4}, \xi=\xi_{1}+\xi_{2}, \tau=\tau_{1}+\tau_{2},\left|\xi_{1}\right| \leq\left|\xi_{2}\right| \leq 18\right\}, \\
\Omega_{2}= & \left\{\left(\xi_{1}, \tau_{1}, \xi, \tau\right) \in \mathbf{R}^{4}, \xi=\xi_{1}+\xi_{2}, \tau=\tau_{1}+\tau_{2},\left|\xi_{2}\right| \geq 18,\left|\xi_{2}\right| \geq 4\left|\xi_{1}\right|,\left|\xi_{1}\right| \leq 1\right\}, \\
\Omega_{3}= & \left\{\left(\xi_{1}, \tau_{1}, \xi, \tau\right) \in \mathbf{R}^{4}, \xi=\xi_{1}+\xi_{2}, \tau=\tau_{1}+\tau_{2},\left|\xi_{2}\right| \geq 18,\left|\xi_{2}\right| \geq 4\left|\xi_{1}\right|,\left|\xi_{1}\right| \geq 1\right\}, \\
\Omega_{4}= & \left\{\left(\xi_{1}, \tau_{1}, \xi, \tau\right) \in \mathbf{R}^{4}, \xi=\sum_{k=1}^{2} \xi_{k}, \tau=\sum_{k=1}^{2} \tau_{k},\left|\xi_{2}\right| \geq 18,\right. \\
& \left.\left|\xi_{1}\right| \leq\left|\xi_{2}\right| \leq 4\left|\xi_{1}\right|,|\xi| \leq \frac{1}{2}\left|\xi_{1}\right|, \xi_{1} \xi_{2} \leq 0\right\}, \\
\Omega_{5}= & \left\{\left(\xi_{1}, \tau_{1}, \xi, \tau\right) \in \mathbf{R}^{4}, \xi=\xi_{1}+\xi_{2}, \tau=\tau_{1}+\tau_{2},\left|\xi_{2}\right| \geq 18,\left|\xi_{1}\right| \leq\left|\xi_{2}\right| \leq 4\left|\xi_{1}\right|,\left|\xi_{2}\right| \geq \frac{|\xi|}{2}\right\}, \\
\Omega_{6}= & \left\{\left(\xi_{1}, \tau_{1}, \xi, \tau\right) \in \mathbf{R}^{4}, \xi=\xi_{1}+\xi_{2}, \tau=\tau_{1}+\tau_{2},\left|\xi_{2}\right| \geq 18,\left|\xi_{1}\right| \leq\left|\xi_{2}\right| \leq 4\left|\xi_{1}\right|, \xi_{1} \xi_{2} \geq 0\right\} .
\end{aligned}
$$

We will denote the integrals in (3.5) corresponding to $\Omega_{k}(1 \leq k \leq 6)$ by $J_{k}(1 \leq k \leq 6)$, respectively. Let $f=\mathscr{F}^{-1} \frac{F}{\langle\sigma\rangle^{-b^{\prime}}}, f_{k}=\mathscr{F}^{-1} \frac{F_{k}}{\left\langle\sigma_{k}\right\rangle^{b}}, k=1,2$. 
(1) Subregion $\Omega_{1}$. Since $\left|\xi_{1}\right| \leq\left|\xi_{2}\right| \leq 18$, we have $|\xi| \leq 36$, which yields

$$
K_{1}\left(\xi_{1}, \tau_{1}, \xi, \tau\right) \leq \frac{C}{\langle\sigma\rangle^{-b^{\prime}} \prod_{k=1}^{2}\left\langle\sigma_{k}\right\rangle^{b}} .
$$

Then, by the Plancherel identity, the Hölder inequality, and $\frac{4}{7} b<b$, we derive

$$
\begin{aligned}
J_{1} & \leq C \int_{\mathbf{R}^{2}} \int_{\substack{\xi=\xi_{1}+\xi_{2} \\
\tau=\tau_{1}+\tau_{2}}} K_{1}\left(\xi_{1}, \tau_{1}, \xi, \tau\right) F \prod_{k=1}^{2} F_{k} d \xi_{1} d \tau_{1} d \xi d \tau \\
& \leq C \int_{\mathbf{R}^{2}} \int_{\substack{\xi=\xi_{1}+\xi_{2} \\
\tau=\tau_{1}+\tau_{2}}} \frac{F \prod_{k=1}^{2} F_{k}}{\langle\sigma\rangle^{-b^{\prime}} \prod_{k=1}^{2}\left\langle\sigma_{k}\right\rangle} d \xi_{1} d \tau_{1} d \xi d \tau \leq C \int_{\mathbf{R}^{2}} f_{1} f_{2} f d x d t \\
& \leq C\|f\|_{L_{x t}^{2}} \prod_{k=1}^{2}\left\|f_{k}\right\|_{L_{x t}^{4}} \leq C\|F\|_{L_{\xi \tau}^{2}} \prod_{k=1}^{2}\left\|f_{k}\right\|_{X_{0, \frac{4}{7} b}} \leq C\|F\|_{L_{\xi \tau}^{2} \prod_{k=1}^{2}\left\|F_{k}\right\|_{L_{\xi \tau}^{2}} .}^{2}
\end{aligned}
$$

(2) Subregion $\Omega_{2}$. In this subregion, obviously, $\left|\xi_{2}\right| \sim|\xi|$.

It is easily checked that

$$
K_{1}\left(\xi_{1}, \tau_{1}, \xi, \tau\right) \leq C \frac{|\xi|^{2}\langle\sigma\rangle^{b^{\prime}}}{\prod_{k=1}^{2}\left\langle\sigma_{k}\right\rangle^{b}} \leq C \frac{\left|\xi_{1}^{6}-\xi_{2}^{6}\right|^{1 / 2}}{\prod_{k=1}^{2}\left\langle\sigma_{k}\right\rangle^{b}}
$$

Consequently, by the Cauchy-Schwarz inequality and Lemma 2.2, we have

$$
\begin{aligned}
J_{2} & \leq C \int_{\mathbf{R}^{2}} \int_{\substack{\xi=\xi_{1}+\xi_{2} \\
\tau=\tau_{1}+\tau_{2}}} K_{1}\left(\xi_{1}, \tau_{1}, \xi, \tau\right) F \prod_{k=1}^{2} F_{k} d \xi_{1} d \tau_{1} d \xi d \tau \\
& \leq C \int_{\mathbf{R}^{2}} \int_{\substack{\xi=\xi_{1}+\xi_{2} \\
\tau=\tau_{1}+\tau_{2}}} \frac{F \prod_{k=1}^{2} F_{k}\left|\xi_{1}^{6}-\xi_{2}^{6}\right|^{\frac{1}{2}}}{\prod_{k=1}^{2}\left\langle\sigma_{k}\right\rangle^{b}} d \xi_{1} d \tau_{1} d \xi d \tau \\
& \leq C\left\|\int_{\substack{\xi=\xi_{1}+\xi_{2} \\
\tau=\tau_{1}+\tau_{2}}} \frac{\left|\xi_{1}^{6}-\xi_{2}^{6}\right|^{\frac{1}{2}} \prod_{k=1}^{2} F_{k}}{\prod_{k=1}^{2}\left\langle\sigma_{k}\right\rangle^{b}} d \xi_{1} d \tau_{1}\right\|_{L_{\xi \tau}^{2}}\|F\|_{L_{\xi \tau}^{2}} \\
& \leq C\|F\|_{L_{\xi \tau}^{2}} \prod_{k=1}^{2}\left\|F_{k}\right\|_{L_{\xi \tau}^{2}} \cdot
\end{aligned}
$$

(3) Subregion $\Omega_{3}$. In this subregion, we derive $|\xi| \sim\left|\xi_{2}\right|$. Thus,

$$
K_{1}\left(\xi_{1}, \tau_{1}, \xi, \tau\right) \leq C \frac{|\xi|^{2}\left|\xi_{1}\right|^{-s}\langle\sigma\rangle^{b^{\prime}}}{\prod_{k=1}^{2}\left\langle\sigma_{k}\right\rangle^{b}}
$$

(i) Case $|\sigma|=\max \left\{|\sigma|,\left|\sigma_{1}\right|,\left|\sigma_{2}\right|\right\}$. By (3.1), we derive

$$
K_{1}\left(\xi_{1}, \tau_{1}, \xi, \tau\right) \leq C \frac{|\xi|^{2+6 b^{\prime}}\left|\xi_{1}\right|^{-s+b^{\prime}}}{\prod_{k=1}^{2}\left\langle\sigma_{k}\right\rangle^{b}} \leq C \frac{\left|\xi_{1}^{6}-\xi_{2}^{6}\right|^{1 / 2}}{\prod_{k=1}^{2}\left\langle\sigma_{k}\right\rangle^{b}}
$$

If $-s+b^{\prime} \leq 0$, then

$$
K_{1}\left(\xi_{1}, \tau_{1}, \xi, \tau\right) \leq C \frac{|\xi|^{2+6 b^{\prime}}\left|\xi_{1}\right|^{-s+b^{\prime}}}{\prod_{k=1}^{2}\left\langle\sigma_{k}\right\rangle^{b}} \leq C \frac{\left|\xi_{1}^{6}-\xi_{2}^{6}\right|^{1 / 2}}{\prod_{k=1}^{2}\left\langle\sigma_{k}\right\rangle^{b}}
$$


If $-s+b^{\prime} \geq 0$, then

$$
K_{1}\left(\xi_{1}, \tau_{1}, \xi, \tau\right) \leq C \frac{|\xi|^{2-s+7 b^{\prime}}}{\prod_{k=1}^{2}\left\langle\sigma_{k}\right\rangle^{b}} \leq C \frac{\left|\xi_{1}^{6}-\xi_{2}^{6}\right|^{1 / 2}}{\prod_{k=1}^{2}\left\langle\sigma_{k}\right\rangle^{b}}
$$

This case can be proved similarly to $\Omega_{2}$.

(ii) Case $\left|\sigma_{1}\right|=\max \left\{|\sigma|,\left|\sigma_{1}\right|,\left|\sigma_{2}\right|\right\}$. Since $\langle\sigma\rangle^{b+b^{\prime}} \leq\left\langle\sigma_{1}\right\rangle^{b+b^{\prime}}$, we have

$$
K_{1}\left(\xi_{1}, \tau_{1}, \xi, \tau\right) \leq C \frac{|\xi|^{2}\left|\xi_{1}\right|^{-s}\left\langle\sigma_{1}\right\rangle^{b^{\prime}}}{\left\langle\sigma_{2}\right\rangle^{b}\langle\sigma\rangle^{b}} \leq C \frac{|\xi|^{2+6 b^{\prime}}\left|\xi_{1}\right|^{-s+b^{\prime}}}{\left\langle\sigma_{2}\right\rangle^{b}\langle\sigma\rangle^{b}}
$$

If $-s+b^{\prime} \leq 0$, we have

$$
K_{1}\left(\xi_{1}, \tau_{1}, \xi, \tau\right) \leq C \frac{\left|\xi_{2}\right|^{5 / 4}}{\left\langle\sigma_{2}\right\rangle^{b}\langle\sigma\rangle^{b}}
$$

consequently, by using the Cauchy-Schwarz inequality and (2.5) and (2.4), we have

$$
\begin{aligned}
& \int_{\mathbf{R}^{2}} \int_{\substack{\xi=\xi_{1}+\xi_{2} \\
\tau=\tau_{1}+\tau_{2}}} \frac{\left|\xi_{2}\right|^{\frac{2 n-1}{4}} F \prod_{j=1}^{2} F_{j}}{\left\langle\sigma_{2}\right\rangle^{b}\langle\sigma\rangle^{b}} d \xi_{1} d \tau_{1} d \xi d \tau \\
& \quad \leq\left\|\langle\sigma\rangle^{-b} \int_{\mathbf{R}^{2}}\left|\xi_{2}\right|^{\frac{5}{4}}\langle\sigma\rangle^{-b} \prod_{j=1}^{2} F_{j} d \xi_{1} d \tau_{1}\right\|_{L_{\xi \tau}^{2}}\|F\|_{L_{\xi \tau}^{2}} \\
& \quad \leq C\left\|\mathscr{F}^{-1}\left(F_{1}\right)\left(D_{x}^{\frac{5}{4}} f_{2}\right)\right\|\left\|_{L_{t}^{\frac{4}{3}} L_{x}^{2}}\right\| F \|_{L_{\xi \tau}^{2}} \\
& \quad \leq C\left\|\mathscr{F}^{-1}\left(F_{1}\right)\right\|_{L_{x t}^{2}}\left\|D_{x}^{5 / 4} f_{2}\right\|_{L_{t}^{4} L_{x}^{\infty}}\|F\|_{L_{\xi \tau}^{2}} \\
& \quad \leq C\left\|F_{1}\right\|_{L_{\xi \tau}^{2}}\|F\|_{L_{\xi \tau}^{2}}\left\|f_{2}\right\|_{X_{0, b}} \\
& \quad \leq C\|F\|_{L_{\xi \tau}^{2} \prod_{j=1}^{2}\left\|F_{j}\right\|_{L_{\xi \tau}^{2}} .}
\end{aligned}
$$

If $-s+b^{\prime} \geq 0$, since $s \geq-\frac{7}{4}+21 \epsilon$, we have

$$
K_{1}\left(\xi_{1}, \tau_{1}, \xi, \tau\right) \leq C \frac{|\xi|^{3-s+7 b^{\prime}}}{\left\langle\sigma_{2}\right\rangle^{b}\langle\sigma\rangle^{b}} \leq C \frac{\left|\xi_{2}\right|^{5 / 4}}{\left\langle\sigma_{2}\right\rangle^{b}\langle\sigma\rangle^{b}}
$$

This case can be proved similarly to the above case.

(iii) Case $\left|\sigma_{2}\right|=\max \left\{|\sigma|,\left|\sigma_{1}\right|,\left|\sigma_{2}\right|\right\}$. This case is similar to (ii) case $\left|\sigma_{1}\right|=\max \left\{|\sigma|,\left|\sigma_{1}\right|\right.$, $\left.\left|\sigma_{2}\right|\right\}$.

(4) Subregion $\Omega_{4}$. In this subregion, $\left|\xi_{1}\right| \sim\left|\xi_{2}\right|$, and it is easy to obtain

$$
\left|\xi_{1}^{6}-\xi_{2}^{6}\right| \geq C|\xi|\left|\xi_{1}\right|^{5}, \quad\left|\xi^{6}-\xi_{1}^{6}\right| \geq C\left|\xi_{1}\right|^{6}, \quad\left|\xi^{6}-\xi_{2}^{6}\right| \geq C\left|\xi_{2}\right|^{6}
$$

(i) Case $|\sigma|=\max \left\{|\sigma|,\left|\sigma_{1}\right|,\left|\sigma_{2}\right|\right\}$. By using, $\left|\xi_{1}\right| \sim\left|\xi_{2}\right|$, when $s \geq 0$, we have

$$
K_{1}\left(\xi_{1}, \tau_{1}, \xi, \tau\right) \leq C \frac{|\xi|^{3}\langle\sigma\rangle^{b^{\prime}}}{\prod_{k=1}^{2}\left\langle\sigma_{k}\right\rangle^{b}} \leq C \frac{\left|\xi_{1}^{6}-\xi_{2}^{6}\right|^{1 / 2}\langle\sigma\rangle^{b^{\prime}}}{\prod_{k=1}^{2}\left\langle\sigma_{k}\right\rangle^{b}}
$$


This case can be proved similarly to Subregion $\Omega_{2}$. When $s \leq 0$, we have

$$
K_{1}\left(\xi_{1}, \tau_{1}, \xi, \tau\right) \leq C \frac{|\xi|^{3}\left|\xi_{1}\right|^{-2 s}}{\langle\sigma\rangle^{-b^{\prime}} \prod_{j=1}^{2}\left\langle\sigma_{j}\right\rangle^{b}}
$$

If $|\sigma|=\max \left\{|\sigma|,\left|\sigma_{1}\right|,\left|\sigma_{2}\right|\right\}$, since $-\frac{7}{4}+21 \epsilon \leq s \leq 0$, then

$$
\begin{aligned}
K_{1}\left(\xi_{1}, \tau_{1}, \xi, \tau\right) & \leq C \frac{|\xi|^{3+b^{\prime}}\left|\xi_{1}\right|^{-2 s+6 b^{\prime}}}{\prod_{k=1}^{2}\left\langle\sigma_{k}\right\rangle^{b}} \\
& \leq C \frac{|\xi|^{1 / 2}\left|\xi_{1}\right|^{5 / 2}|\xi|^{\frac{5}{2}+b^{\prime}}\left|\xi_{1}\right|^{-2 s+6 b^{\prime}-5 / 2}}{\prod_{k=1}^{2}\left\langle\sigma_{k}\right\rangle^{b}} \\
& \leq C \frac{\left|\xi_{1}^{6}-\xi_{2}^{6}\right|^{1 / 2}}{\prod_{k=1}^{2}\left\langle\sigma_{k}\right\rangle^{b}}
\end{aligned}
$$

By using the Cauchy-Schwarz inequality, we have

$$
\begin{aligned}
J_{4} & \leq C \int_{\mathbf{R}^{2}} \int_{\substack{\xi=\xi_{1}+\xi_{2} \\
\tau=\tau_{1}+\tau_{2}}} K_{1}\left(\xi_{1}, \tau_{1}, \xi, \tau\right) F \prod_{k=1}^{2} F_{k} d \xi_{1} d \tau_{1} d \xi d \tau \\
& \leq C \int_{\mathbf{R}^{2}} \int_{\substack{\xi=\xi_{1}+\xi_{2} \\
\tau=\tau_{1}+\tau_{2}}} \frac{\left|\xi_{1}^{6}-\xi_{2}^{6}\right|^{1 / 2}}{\prod_{k=1}^{2}\left\langle\sigma_{k}\right\rangle^{b}} F \prod_{k=1}^{2} F_{k} d \xi_{1} d \tau_{1} d \xi d \tau \\
& \leq C\left\|\int_{\substack{\xi=\xi_{1}+\xi_{2} \\
\tau=\tau_{1}+\tau_{2}}} \frac{\left|\xi_{1}^{6}-\xi_{2}^{6}\right|^{1 / 2}}{\prod_{k=1}^{2}\left\langle\sigma_{k}\right\rangle^{b}} \prod_{k=1}^{2} F_{k} d \xi_{1} d \tau_{1}\right\|_{L_{\xi \tau}^{2}}\|F\|_{L_{\xi \tau}^{2}} \\
& \leq C\|F\|_{L_{\xi \tau}^{2}} \prod_{j=1}^{2}\left\|F_{j}\right\|_{L^{2} .}
\end{aligned}
$$

(ii) Case $\max \left\{|\sigma|,\left|\sigma_{1}\right|,\left|\sigma_{2}\right|\right\}=\left|\sigma_{1}\right|$. Since $\langle\sigma\rangle^{b+b^{\prime}} \leq\left\langle\sigma_{1}\right\rangle^{b+b^{\prime}}$, by using $-\frac{7}{4}+21 \epsilon \leq s \leq 0$, we have

$$
K_{1}\left(\xi_{1}, \tau_{1}, \xi, \tau\right) \leq C \frac{|\xi|^{3}\left|\xi_{1}\right|^{-2 s}}{\langle\sigma\rangle^{b}\left\langle\sigma_{2}\right\rangle^{b}\left\langle\sigma_{1}\right\rangle^{-b^{\prime}}} \leq C \frac{|\xi|^{3+b^{\prime}}\left|\xi_{1}\right|^{-2 s+6 b^{\prime}}}{\langle\sigma\rangle^{b}\left\langle\sigma_{2}\right\rangle^{b}} \leq C \frac{\left|\xi^{6}-\xi_{2}^{6}\right|^{1 / 2}}{\langle\sigma\rangle^{b}\left\langle\sigma_{2}\right\rangle^{b}}
$$

This case can be proved similarly to $\max \left\{|\sigma|,\left|\sigma_{1}\right|,\left|\sigma_{2}\right|\right\}=|\sigma|$.

(iii) Case $\max \left\{|\sigma|,\left|\sigma_{1}\right|,\left|\sigma_{2}\right|\right\}=\left|\sigma_{2}\right|$.

This case can be proved similarly to $\max \left\{|\sigma|,\left|\sigma_{1}\right|,\left|\sigma_{2}\right|\right\}=\left|\sigma_{1}\right|$.

(5) Subregion $\Omega_{5}$. In this region $|\xi| \sim\left|\xi_{1}\right| \sim\left|\xi_{2}\right|$, thus, we have

$$
K_{1}\left(\xi_{1}, \tau_{1}, \xi, \tau\right) \leq C \frac{|\xi|^{3-s}\langle\sigma\rangle^{b^{\prime}}}{\prod_{k=1}^{2}\left\langle\sigma_{k}\right\rangle^{b}}
$$

(i) If $|\sigma|=\max \left\{|\sigma|,\left|\sigma_{1}\right|,\left|\sigma_{2}\right|\right\}$, by using (3.1) and $s \geq-\frac{9}{4}+\frac{21}{2} \epsilon$, we have

$$
K_{1}\left(\xi_{1}, \tau_{1}, \xi, \tau\right) \leq C \frac{|\xi|^{3-s+7 b^{\prime}}}{\prod_{k=1}^{2}\left\langle\sigma_{k}\right\rangle^{b}} \leq C \frac{\prod_{k=1}^{2}\left|\xi_{k}\right|^{\frac{5}{8}}}{\prod_{k=1}^{2}\left\langle\sigma_{k}\right\rangle^{b}}
$$


By using the Plancherel identity, the Hölder inequality, and $\frac{3}{4} b<b$ as well as (2.5), we have

$$
\begin{aligned}
J_{5} & \leq C \int_{\mathbf{R}^{2}} \int_{\substack{\xi=\xi_{1}+\xi_{2} \\
\tau=\tau_{1}+\tau_{2}}} K_{1}\left(\xi_{1}, \tau_{1}, \xi, \tau\right) F \prod_{k=1}^{2} F_{k} d \xi_{1} d \tau_{1} d \xi d \tau \\
& \leq C \int_{\mathbf{R}^{2}} \int_{\substack{\xi=\xi_{1}+\xi_{2} \\
\tau=\tau_{1}+\tau_{2}}} \frac{\prod_{k=1}^{2}\left|\xi_{k}\right|^{\frac{5}{8}}}{\prod_{k=1}^{2}\left\langle\sigma_{k}\right\rangle^{b}} d \xi_{1} d \tau_{1} d \xi d \tau \\
& \leq C \int_{\mathbf{R}^{2}} \mathscr{F}^{-1} F \prod_{k=1}^{2} D_{x}^{\frac{5}{8}} f_{k} d x d t \\
& \leq C\left\|\mathscr{F}^{-1} F\right\|_{L_{x t}^{2}} \prod_{k=1}^{2}\left\|D_{x}^{\frac{5}{8}} f_{k}\right\|_{L_{x t}^{4}} \\
& \leq C\|F\|_{L_{\xi \tau}^{2}} \prod_{k=1}^{2}\left\|f_{k}\right\|_{X_{0, \frac{3}{4} b}} \leq C\|F\|_{L_{\xi \tau}^{2}} \prod_{k=1}^{2}\left\|F_{k}\right\|_{L_{\xi \tau}^{2}} .
\end{aligned}
$$

(ii) If $\left|\sigma_{1}\right|=\max \left\{|\sigma|,\left|\sigma_{1}\right|,\left|\sigma_{2}\right|\right\}$, then $\langle\sigma\rangle^{b^{\prime}}\left\langle\sigma_{1}\right\rangle^{-b} \leq\left\langle\sigma_{1}\right\rangle^{b^{\prime}}\langle\sigma\rangle^{-b}$. By using (3.1), we have

$$
K_{2}\left(\xi_{1}, \tau_{1}, \xi, \tau\right) \leq C \frac{|\xi|^{2-s}\left\langle\sigma_{1}\right\rangle^{b^{\prime}}}{\left\langle\sigma_{2}\right\rangle^{b}\langle\sigma\rangle^{b}} \leq C \frac{|\xi|^{2-s+7 b^{\prime}}}{\left\langle\sigma_{2}\right\rangle^{b}\langle\sigma\rangle^{b}} \leq C \frac{|\xi|^{\frac{5}{8}}\left|\xi_{2}\right|^{\frac{5}{8}}}{\langle\sigma\rangle^{b}\left\langle\sigma_{2}\right\rangle^{b}}
$$

By using the Plancherel identity, the Hölder inequality, (2.5) and $\frac{3}{4} b<b$, we have

$$
\begin{aligned}
& J_{5} \leq C \int_{\mathbf{R}^{2}} \int_{\substack{\xi=\xi_{1}+\xi_{2} \\
\tau=\tau_{1}+\tau_{2}}} K_{1}\left(\xi_{1}, \tau_{1}, \xi, \tau\right) F \prod_{k=1}^{2} F_{k} d \xi_{1} d \tau_{1} d \xi d \tau \\
& \leq C \int_{\mathbf{R}^{2}} \int_{\substack{\xi=\xi_{1}+\xi_{2} \\
\tau=\tau_{1}+\tau_{2}}} \frac{|\xi|^{\frac{5}{8}}\left|\xi_{2}\right|^{\frac{5}{8}}}{\left\langle\sigma_{2}\right\rangle^{b}\left\langle\sigma_{2}\right\rangle^{b}} F \prod_{k=1}^{2} F_{k} d \xi_{1} d \tau_{1} d \xi d \tau \\
& \leq C \int_{\mathbf{R}^{2}}\left(\mathscr{F}^{-1} F_{1}\right)\left(D_{x}^{\frac{5}{8}} \mathscr{F}^{-1}\left(\frac{F}{\langle\sigma\rangle^{b}}\right)\right) D_{x}^{\frac{5}{8}} f_{2} d x d t \\
& \leq C\left\|\mathscr{F}^{-1} F_{1}\right\|_{L_{x t}^{2}}\left\|D_{x}^{\frac{5}{8}} f_{2}\right\|_{L_{x t}^{4}}\left\|D_{x}^{\frac{5}{8}} \mathscr{F}^{-1}\left(\frac{F}{\langle\sigma\rangle^{b}}\right)\right\|_{L_{x t}^{4}} \\
& \leq C\left\|F_{1}\right\|_{L_{\xi \tau}^{2}}\left\|f_{2}\right\|_{X_{0, \frac{3}{4} b} b}\left\|\frac{F}{\langle\sigma\rangle^{b}}\right\|_{X_{0, \frac{3}{4} b}} \leq C\|F\|_{L_{\xi \tau}^{2}} \prod_{k=1}^{2}\left\|F_{k}\right\|_{L_{\xi \tau}^{2}} .
\end{aligned}
$$

(iii) If $\left|\sigma_{2}\right|=\max \left\{|\sigma|,\left|\sigma_{1}\right|,\left|\sigma_{2}\right|\right\}$.

This case can be proved similarly to the case $\left|\sigma_{1}\right|=\max \left\{|\sigma|,\left|\sigma_{1}\right|,\left|\sigma_{2}\right|\right\}$.

(6) Subregion $\Omega_{6}$. In this region, we have $|\xi| \sim\left|\xi_{1}\right| \sim\left|\xi_{2}\right|$.

This case can be proved similarly to the Subregion $\Omega_{5}$.

We have completed the proof of Lemma 3.1.

\section{Proof of Theorem 1.1}

The system (1.1)-(1.2) is equivalent to the following integral equation:

$$
u(t)=W(t) u_{0}+\int_{0}^{t} W(t-\tau) \partial_{x}^{2}\left(u^{2}\right) d \tau .
$$


We define

$$
\Phi(u)=\Psi(t) W(t) u_{0}+\Psi_{\delta}(t) \int_{0}^{t} W(t-\tau) \partial_{x}^{2}\left(u^{2}\right) d \tau
$$

Combining Lemmas 2.3 and 3.1 with the fixed point theorem, we easily obtain Theorem 1.1.

\section{Competing interests}

The authors declare that they have no competing interests.

Authors' contributions

All authors read and approved the final manuscript.

\section{Author details}

${ }^{1}$ College of Mathematics and Information Science, Henan Normal University, Xinxiang, Henan 453007, P.R. China. ${ }^{2}$ Henan Vocational College of Agriculture, Zhengzhou, Henan 451450, P.R. China.

\section{Acknowledgements}

We would like to thank reviewers for a careful reading and valuable comments on the original draft. The first author is supported by Foundation and Frontier of Henan Province under grant Nos. 122300410414, 132300410432.

Received: 5 March 2014 Accepted: 6 May 2014 Published: 20 May 2014

\section{References}

1. Kenig, CE, Ponce, G, Vega, L: Higher-order nonlinear dispersive equations. Proc. Am. Math. Soc. 122, 157-166 (1994)

2. Pilod, D: On the Cauchy problem for higher-order nonlinear dispersive equations. J. Differ. Equ. 245, 2055-2077 (2008)

3. Guo, ZH, Kwak, C, Kwon, S: Rough solutions of the fifth-order KdV equations. J. Funct. Anal. 265, 2791-2829 (2013)

4. Bourgain, J: Fourier transform restriction phenomena for certain lattice subsets and applications to nonlinear evolution equations, part II: the KdV equation. Geom. Funct. Anal. 3, 209-262 (1993)

5. Li, Y, Yan, W, Yang, $X:$ Well-posedness of a higher order modified Camassa-Holm equation in spaces of low regularity. J. Evol. Equ. 10, 465-486 (2010)

6. Li, Y, Li, S, Yan, W: Sharp well-posedness and ill-posedness of a higher-order modified Camassa-Holm equation. Differ. Integral Equ. 25, 1053-1074 (2012)

10.1186/1687-2770-2014-122

Cite this article as: Wang and Zheng: The Cauchy problem for the seventh-order dispersive equation in Sobolev space. Boundary Value Problems 2014, 2014:122

\section{Submit your manuscript to a SpringerOpen ${ }^{\circ}$ journal and benefit from:}

- Convenient online submission

- Rigorous peer review

Immediate publication on acceptance

Open access: articles freely available online

- High visibility within the field

- Retaining the copyright to your article 